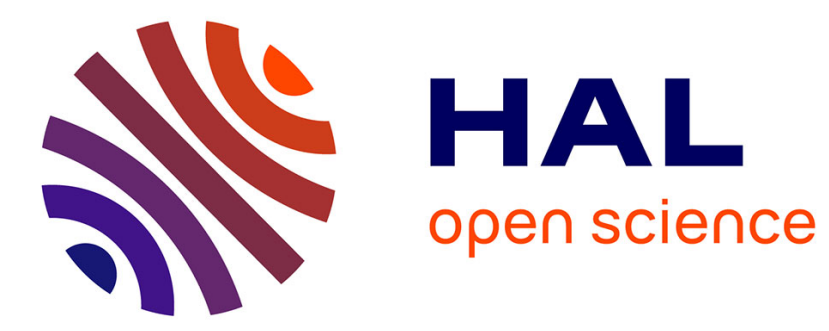

\title{
Langues nationales et régionales : une relation géopolitique
}

Barbara Loyer

\section{To cite this version:}

Barbara Loyer. Langues nationales et régionales: une relation géopolitique. Langues et territoires, Hérodote $\mathrm{n}^{\circ} 105$, pp. 15-37, 2002. hal-00705705

\section{HAL Id: hal-00705705 \\ https://hal.science/hal-00705705}

Submitted on 23 Mar 2019

HAL is a multi-disciplinary open access archive for the deposit and dissemination of scientific research documents, whether they are published or not. The documents may come from teaching and research institutions in France or abroad, or from public or private research centers.
L'archive ouverte pluridisciplinaire HAL, est destinée au dépôt et à la diffusion de documents scientifiques de niveau recherche, publiés ou non, émanant des établissements d'enseignement et de recherche français ou étrangers, des laboratoires publics ou privés. 


\title{
LANGUES NATIONALES ET RÉGIONALES : UNE RELATION GÉOPOLITIQUE
}

Barbara Loyer

\author{
La Découverte | «Hérodote»
}

2002/2 $\mathrm{N}^{\circ} 105$ | pages 15 à 37

ISSN 0338-487X

ISBN 270713537X

Article disponible en ligne à l'adresse :

https://www.cairn.info/revue-herodote-2002-2-page-15.htm

Distribution électronique Cairn.info pour La Découverte.

(C) La Découverte. Tous droits réservés pour tous pays.

La reproduction ou représentation de cet article, notamment par photocopie, n'est autorisée que dans les limites des conditions générales d'utilisation du site ou, le cas échéant, des conditions générales de la licence souscrite par votre établissement. Toute autre reproduction ou représentation, en tout ou partie, sous quelque forme et de quelque manière que ce soit, est interdite sauf accord préalable et écrit de l'éditeur, en dehors des cas prévus par la législation en vigueur en France. Il est précisé que son stockage dans une base de données est également interdit. 


\title{
Langues nationales et régionales : une relation géopolitique
}

\author{
Barbara Loyer*
}

Du point de vue de la Charte européenne des langues régionales ou minoritaires, la reconnaissance du plus grand nombre d'idiomes en Europe est frappée du sceau de la justice et ne peut donc avoir d'effets négatifs sur les sociétés où elle s'applique. Selon ses rédacteurs, la diversité des situations nationales est prise en compte par le fait que, dans chaque domaine - enseignement, médias, justice, administration publique...-, des articles de portées très différentes sont proposés à la ratification, de sorte que chaque État puisse doser le degré d'existence qu'il souhaite accorder aux langues minoritaires. Mais ce n'est pas suffisant pour éliminer toutes les réticences que soulève la ratification de la Charte (seize ratifications sur quarante-trois membres du Conseil de l'Europe depuis 1992) parce que, bien souvent, le plurilinguisme sur un territoire est source de conflit ou s'intègre dans des rivalités plus complexes. À cet égard, le cas de l'Espagne et celui de la France sont intéressants à comparer car ce sont deux très anciens États confrontés à des revendications linguistiques multiples. En 1978, après la dictature franquiste, l'Espagne a reconnu la co-officialité du catalan, du basque, du galicien avec l'espagnol. Néanmoins, les antagonismes à ce sujet n'ont pas disparu et se sont même, dans le cas basque, exacerbés. L'analyse des langues d'un point de vue géopolitique situe ces conflits dans un contexte plus large que leur seule dimension linguistique. Elle s'attache également à décrire les représentations contradictoires dont ils font l'objet, c'est-à-dire les différentes manières de présenter les faits selon l'opinion que l'on défend.

* Géographe, université Paris-VIII. 


\section{En France, l'enseignement des langues régionales se développe}

En France, la personnification de l'État présenté comme un être luttant pour ne rien céder de ses prérogatives, une sorte de roi d'Ancien Régime, est assez courante. Pourtant, cette représentation est un écran qui empêche d'examiner les multiples facettes de la réalité. En ce qui concerne l'enseignement, en effet, le renouvellement des hommes et des idées qu'a permis l'arrivée des socialistes au pouvoir en 1981 a été le point de départ d'un mouvement en faveur de l'enseignement des langues régionales, notamment à partir de la circulaire de $1982^{1}$ du ministre de l'Éducation nationale Alain Savary. Plusieurs académies de l'Éducation nationale ont mis sur pied des systèmes d'enseignement bilingue françaislangue régionale qui sont maintenant en plein essor. Au total, d'après les statistiques du ministère de l'Éducation nationale, l'enseignement bilingue a progressé, entre 1998 et 2001, de 4071 élèves pour l'école et de 2463 élèves pour le collège. Les effectifs affichés sont de 2868 élèves en occitan langue d'oc, 839 en catalan, 6099 en basque, 6592 en breton, 7323 dans les langues d'Alsace et 5823 pour les langues mosellanes ${ }^{2}$.

Au sein des sections bilingues, les enfants étudient la moitié des matières en français et l'autre moitié, en général les disciplines scientifiques, dans la langue régionale. Les premières ouvertures de ce type ont eu lieu sous l'impulsion des recteurs et d'inspecteurs d'académie. Au Pays basque, le recteur Jean-Claude Martin avait fait appel à deux inspectrices, Claudine Leralu et Isabelle Lichau, pour apporter une réponse à la mobilisation locale croissante en faveur des écoles de l'association Seaska («berceau» en euskara). Cette association est membre de la confédération réunissant les ikastolas («école» en euskara) de France et d'Espagne. Elle pratique la méthode de l'immersion totale, c'est-à-dire que toute la vie scolaire se déroule en euskara. À l'époque, Seaska était quasiment, avec quelques écoles catholiques privées, le seul lieu où l'on pouvait faire étudier l'euskara aux enfants. Depuis 1983, l'État français a subventionné Seaska par divers canaux (aujourd'hui, ses enseignants - environ 150 pour 1850 élèves sont payés par le ministère de l'Éducation nationale), mais les deux inspectrices ont proposé une autre forme d'enseignement de la langue basque. Isabelle Lichau

1. $B O \mathrm{n}^{\circ} 26,1^{\mathrm{er}}$ juillet 1982. La circulaire énumère diverses possibilités d'intervention en langue régionale dans les classes. Pour l'école élémentaire, une phrase concerne l'enseignement bilingue: «Enfin, seront étudiées les conditions dans lesquelles pourraient être créées des classes expérimentales bilingues tenant compte des expériences déjà engagées dans certaines régions et faisant appel aux compétences qu'elles ont ainsi révélées. » Le $B O \mathrm{n}^{\circ} 16 \mathrm{du}$ 20 avril 1995 définit beaucoup plus longuement les modalités de l'enseignement bilingue dans le service public.

2. http ://www.education.gouv.fr/discours/2001/rentree/ecole.htm. Ces chiffres englobent apparemment toutes les offres d'enseignement bilingue. 
avait une expérience personnelle de cette question; sa famille s'exprimait en béarnais, et elle a d'abord appris le français à l'école. La filière bilingue qu'elles ont organisée se fonde sur deux principes: respect des programmes nationaux et parité des deux langues. La première section s'est ouverte en maternelle dès 1982, et en primaire l'année suivante. Au début, cette innovation a été critiquée à la fois par les personnes qui craignaient le nationalisme basque ou l'affaiblissement de l'école publique et par Seaska, qui perdait sa place de défenseur exclusif de l'identité locale. Le projet a en revanche reçu le soutien d'élus qui souhaitaient justement éviter que Seaska ou les nationalistes basques aient le monopole de cette identité. Avant la mise en place de la filière bilingue, les premiers enseignants itinérants de langue basque sur le département avaient d'ailleurs été nommés, en $1969^{3}$, par le ministère de l'Éducation nationale sur la demande de personnalités locales, notamment le député de Saint-Jean-Pied-de-Port, Michel Inchauspe (RPR).

À partir de 1986, une association de parents d'élèves des classes bilingues, Ikas Bi (ikas signifie «apprendre» et bi signifie «deux»), qui comprenait au départ bon nombre d'enseignants dans ses rangs, a travaillé à la divulgation des résultats de l'expérience, puis a édité le premier manuel de mathématiques en euskara utilisé en France. La collaboration entre les services de l'académie et Ikas $\mathrm{Bi}$ a été très étroite. Les uns et les autres se reconnaissent une dette mutuelle pour le démarrage de ces classes aménagées. Actuellement, toutes les demandes d'ouverture sont honorées. Le ministère prévoit d'ouvrir quatorze sections supplémentaires en 2003. L'expérience du bilinguisme a ensuite fait des émules pour l'occitan (1989), l'allemand (1992), le catalan (1996). En Alsace, dans les années quatre-vingt, le recteur Deyon avait lui aussi mis en place le mouvement en faveur de l'enseignement bilingue, mais c'est sous l'impulsion du recteur Jean-Paul de Gaudemar, chargé de cette question auprès de Jack Lang, que le mouvement s'est accéléré. On est passé de 10 classes bilingues en 1992 à 218 en 1998-1999, accueillant près de 5000 élèves. L'académie de Strasbourg évalue régulièrement le niveau global des enfants de ces sections et publie systématiquement ses rapports d'évaluation. En outre, elle propose un «cursus universitaire binational sur deux années, suivi de préparation à l'IUFM et à la Pädagogische Hoschschule, du concours de recrutement des professeurs des écoles et du Staatsexamen ( $1^{\text {re }}$ année d'IUFM), et enfin de la formation bilingue ( $2^{\mathrm{e}}$ année d'IUFM) ${ }^{4} \gg$.

En Bretagne, en revanche, le bilinguisme à l'école publique s'est développé essentiellement à l'initiative d'une association de parents d'élèves, Div Yezh

3. Année de la fondation de la première maternelle de Seaska.

4. Daniel MORGEN (directeur adjoint de l'IUFM d'Alsace et directeur du centre de formation aux enseignements bilingues de Guebwiller), Les Nouveaux Cahiers d'allemand, $\mathrm{n}^{\circ}$ spécial, juin 1999. 
(«deux langues », prononcer diou yéz), fondée en 1979, et sans le soutien du rectorat. Div Yezh a également mis à profit la circulaire Savary pour obtenir l'ouverture des premières classes bilingues. Le système est différent : ce sont les mêmes enseignants qui passent du français au breton selon les matières ou les moments de la journée. Il y a, en 2001, 45 écoles offrant cette option à 2649 enfants 5 .

Le fait qu'en octobre 2001 le Conseil d'État ait bloqué l'intégration dans l'Éducation nationale de l'école Diwan (diwan signifie «germe» en breton), qui pratique le système d'immersion totale de l'enfant dans un environnement scolaire en langue bretonne, ne signifie pas que rien ne bouge et ne bougera jamais en France, mais que la question des langues régionales soulève de nombreuses interrogations. Ces questions sont éminemment géopolitiques en ce sens que les revendications linguistiques débouchent sur une remise en cause des représentations traditionnelles de la France comme nation unitaire, ou comme république dont l'homogénéité garantit l'exercice des libertés individuelles. Aux revendications linguistiques sont liées non seulement des opinions différentes au sujet de la liberté individuelle, mais également d'autres représentations du territoire français. Une analyse géopolitique des rivalités linguistiques prend en compte ces images du territoire, que l'on peut cartographier.

\section{Les cartes sont parfois des arguments géopolitiques : l'exemple de la Navarre}

Les partisans de la diffusion des langues minoritaires dissocient en général leurs réclamations de toute référence aux territoires car, pour obtenir les moyens qu'ils demandent, ils évitent de parler de ce qui pourrait être source de conflit. Mais c'est une dimension importante du sujet. L'exemple basque illustre nettement cet aspect du problème car la nation basque revendique une partie du territoire de deux nations, l'espagnole et la française. Côté espagnol, c'est en Navarre que la rivalité se voit le mieux.

La Navarre est une région autonome que les nationalistes basques veulent voir intégrer dans la région autonome basque, parce qu'ils pensent qu'elle fait partie de la nation historique dont ils veulent obtenir l'indépendance. Mais ils n'y représentent pour l'instant qu'un électeur sur cinq (21\% des suffrages exprimés en 1999). L'opposition entre les régionalistes navarrais majoritaires, qui se disent espagnols, et les nationalistes basques est très forte et se cristallise pour une part sur la question linguistique. Le recensement de 1991 dénombre $16 \%$ de bascophones, et cette proportion s'élevait à $73 \%$ de la population dans le Nord, la zone bascophone où la langue basque est co-officielle avec le castillan. Dans la vallée de

\section{5. http ://www.bretagnet.com/div_yezh/}


l'Èbre, au sud, les bascophones représentaient à cette date 2,8\% de la population. En 1986 a été votée une loi, la Ley del Vascuence (loi de la langue basque), qui régissait les droits des locuteurs en euskara. À l'époque, la méfiance entre nationalistes et non-nationalistes basques était moindre, et les socialistes, étant arrivés au pouvoir avec seulement $28 \%$ des suffrages devant la droite régionaliste (UPN $24,8 \%$ ), avaient besoin des voix nationalistes basques pour gouverner. Les compromis se sont faits notamment sur la loi linguistique. Sa première version mettait en place une discrimination positive envers l'euskara. Il fallait connaître cette langue pour avoir accès à certaines places de fonctionnaires dans la zone bascophone, et l'euskara apportait des points supplémentaires à celui qui postulait pour un emploi de ce type dans les deux autres zones. En 1991, l'UPN remporta les élections et le nouveau président, Juan Cruz Alli, poursuivit la politique d'ouverture à l'égard de l'euskara. Il voulut faire passer l'idée qu'il valait mieux intégrer la dimension basque de l'identité navarraise parce qu'une telle identité singulière serait un atout dans ce qu'il appelait le «nouvel ordre européen postmoderne». En effet, d'après lui, de grandes régions s'imposeront face aux Etats, qui ne devraient plus être les dépositaires exclusifs de la souveraineté sur les territoires régionaux ${ }^{6}$. En 1994, il amenda considérablement la loi linguistique dans le sens d'une meilleure protection légale des intérêts des bascophones et fit des gestes concrets en faveur des ikastolas.

Ces innovations, et d'autres, ne furent pas admises par le parti qui, en 1995, choisit un autre candidat à la présidence régionale (Juan Cruz Alli quitte alors UPN pour prendre la tête d'un nouveau parti, Convergencia de Demócratas Navarros). L'idée qui prévaut depuis dans l'UPN est que la discrimination favorable aux locuteurs bascophones a entrainé une surreprésentation des nationalistes basques au sein de l'administration régionale, ceux-ci ayant pu ainsi obtenir des postes clés. C'est pourquoi l'équipe actuellement au pouvoir (UPN), avec l'appui du Parti socialiste, a modifié la loi, en décembre 2001, pour diminuer les contraintes de l'administration navarraise à l'égard de la langue basque et augmenter les possibilités de contrôle sur son usage public. Dans la zone bascophone, elle permet, par exemple, de demander aux autorités locales qu'elles justifient l'exigence de l'euskara pour certains postes. Quant aux emplois pour lesquels la maîtrise du basque apporte des points supplémentaires, il est dit, dans la loi de janvier 2001, que ces points ne peuvent pas représenter plus de $10 \%$ de la note globale, alors qu'en 1994 ces points de bonus ne devaient pas représenter moins de $10 \%$ de la note globale. Enfin, le gouvernement de Pampelune a décidé d'examiner les livres de textes utilisés dans les ikastolas. Les ayant trouvés trop nationalistes, parce que leur principal cadre de référence est la carte des sept

6. Discours d'ouverture du $2^{\mathrm{e}}$ congrès «Culture européenne» de l'université de Navarre, 28 octobre 1992. 
territoires de la nation basque accompagnée d'une réécriture, nationaliste basque, de l'histoire du royaume de Navarre et de l'Espagne, il envisage d'imposer les manuels reconnus par le gouvernement régional, traduits en basque. C'est la bataille actuelle.

L'une des caractéristiques de ce combat linguistique est donc ici de vouloir faire coïncider par une relation d'appartenance historique une langue et un territoire, sans tenir compte des locuteurs qui y viennent ou qui s'en vont. En Navarre, malgré le respect des droits des bascophones au début des années quatre-vingt, l'affrontement entre nationalistes et non-nationalistes basques s'est durci parce que la langue est l'instrument d'un autre combat, celui de la reconnaissance d'un territoire symbolique. En France, l'euskara donne aussi l'occasion d'évoquer une nation basque qui se superpose au territoire de la nation française. Un petit groupe de militants nationalistes basques mobilisés peut entraîner les débats sur une voie assimilant la juxtaposition de la langue basque et de la langue française à la confrontation de deux nations irréductibles. Il est tout à fait possible d'imposer cette seule approche de la question linguistique bien que la majorité des électeurs votent pour des partis qui soutiennent l'enseignement bilingue, mais n'accordent pas pour autant la même valeur symbolique à l'euskara.

Un territoire symbolique n'est cependant pas forcément national; il peut être régional. Ce n'est pas parce que l'on décrit les représentations territoriales liées à des revendications en faveur d'une langue minoritaire que l'on voit du séparatisme dans ces revendications. Même si l'on pense qu'il n'y aura pas de conflit grave entre locuteurs, ces représentations sont un élément de l'analyse dès lors qu'elles font partie des arguments utilisés pour favoriser la pratique d'une langue ou d'une autre. L'uniformité du territoire français est une représentation fondée sur une part de réalité que l'on choisit de favoriser. Elle a été un dogme durant certaines époques, pour des raisons diverses, et a été diffusée par les cartes qui figurent dans les manuels d'histoire-géographie des programmes nationaux. Elle est aujourd'hui relativisée par d'autres représentations qui peuvent devenir aussi des dogmes aux yeux de leurs partisans.

L'ensemble spatial des langues celtes, par exemple, est l'une de ces représentations. Il a été baptisé d'un néologisme, la Celtie, qui regroupe l'Écosse, le Pays de Galles, l'Irlande, la Cornouaille, la Bretagne. Pour voir ce que cette représentation contient, il faut changer de niveau d'analyse et étudier chacune de ses composantes: Hérodote a déjà publié un article sur le sujet ${ }^{7}$. La Bretagne elle-même est une représentation complexe puisque les limites du duché de Bretagne englobaient un territoire qui allait jusqu'à Nantes, aujourd'hui capitale de la région Pays de la Loire. La directrice de l'école Diwan de Landerneau, Ana Vari Chapalain, affirme que l'ouverture des écoles Diwan dans des zones où l'on parlait autrefois

7. $\mathrm{N}^{\circ} 95,4^{\mathrm{e}}$ trimestre 1999, « Nationalismes régionaux en Europe ». 
UNE REPRÉSENTATION DU PAYS BASQUE

\section{DANS UN MANUEL DE L'ENSEIGNEMENT SECONDAIRE}

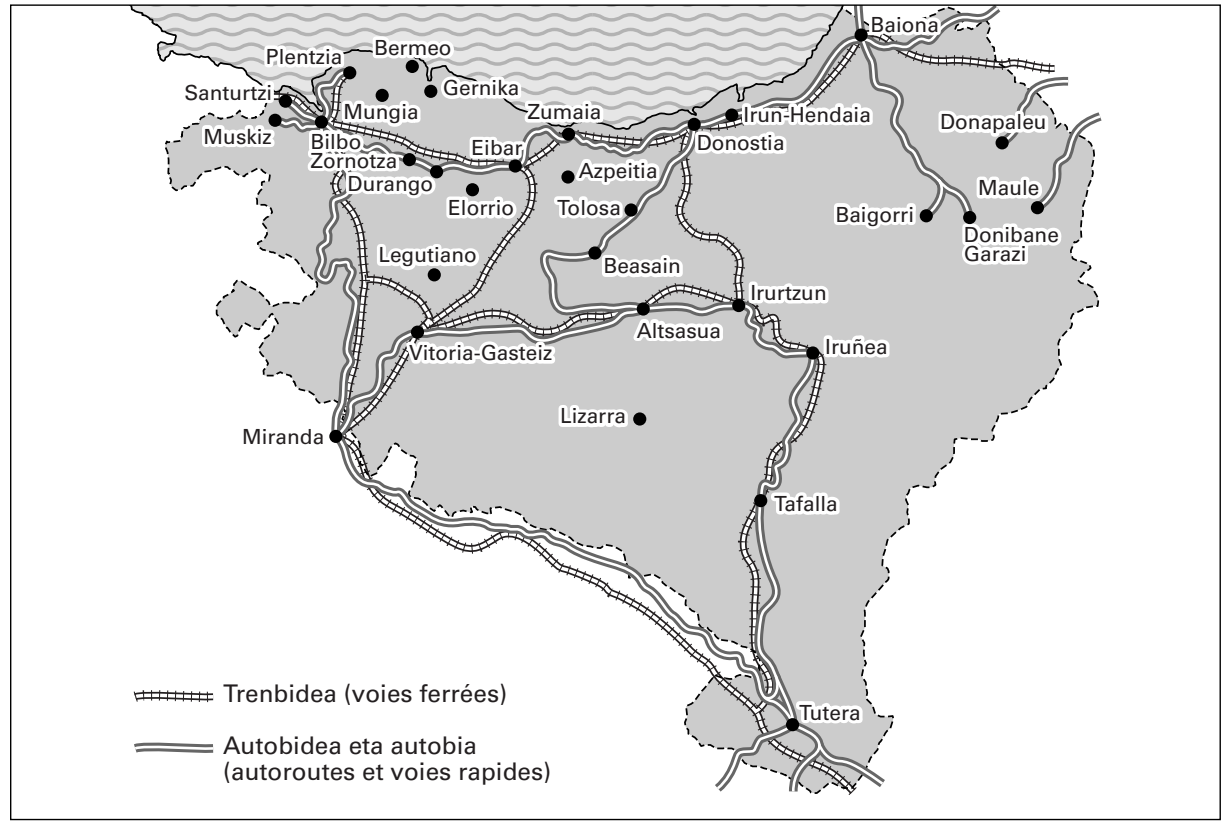

La frontière entre la France et l'Espagne n'apparaît pas (les villes de Baiona, Hendaia, Baigorri, Maule, Donibane, Garazi, Donapaleu sont en France).

Les frontières entre les provinces basques n'apparaissent pas. La Navarre n'est pas individualisé (les villes de Iruñea, Irurtzun, Altsasua, Lizarra, Tafalla, Tutera sont en Navarre).

Les toponymes sont en basque. Iruñea: Pampelune; Donostia : Saint-Sébastien; Baiona: Bayonne; Donibane Garazi : Saint-Jean-Pied-de-Port ; Hendaia : Hendaye; Maule : Mauleon; Donapaleu : Saint-Palais.

Source: Gizarte Zientziak, Geografia eta Historia, Erein Proiektua, 1997.

le gallo et non le breton, à Saint-Nazaire ou à Rennes, ne relève pas d'une stratégie territoriale mais répond à une demande. Diwan a été à une époque sollicité pour enseigner le breton en banlieue parisienne, mais le contexte local ne se prêtait pas, dit-elle, à l'enseignement par immersion, alors qu'en Loire-Atlantique il s'y prête. Cela met en valeur le lien étroit entre les représentations territoriales historiques et l'implantation des cours de langues régionales. Il existe d'ailleurs une réelle volonté de diffuser, par la cartographie des lieux où l'on dispense des cours de langue bretonne dans les limites de l'ancien duché, une image de ce contexte que l'on dit favorable à l'immersion. Cette image permet d'argumenter la nécessité de poursuivre l'effort dans les interstices des implantations existantes, afin de 
compléter l'infrastructure linguistique d'un espace (le mot «espace» est neutre, celui de «territoire» contient l'idée d'appropriation). Il ne s'agit pas de dire ici que la carte est vraie ou fausse. Il y a sans doute des parents habitant en LoireAtlantique qui voudraient faire bénéficier leurs enfants de cours en breton. L'association Div Yezh demande aussi l'ouverture de classes dans ce département (l'inspection académique ne les a pas accordées pour des raisons diverses). Mais les cartes ne sont pas des documents plus objectifs que d'autres. Outre les répartitions qu'elles donnent à voir, elles sont le support de messages, représentations, convictions, qui font partie de l'information.

\section{La grande influence des petits espaces}

Si la France ou l'Espagne apparaissent dans leur complexité dès que l'on s'intéresse aux régions ou aux nationalités qui les composent, il en va de même desdites régions. Bretagne, Pays basque, Catalogne ne sont pas non plus des ensembles simples. Pour les mettre en valeur, il est utile de distinguer différents niveaux d'analyse de la réalité. Plus on prend en considération des ensembles spatiaux vastes, plus on néglige les diversités locales au profit des représentations. Pour repérer et étudier la complexité des problèmes linguistiques, et les décrire, il faut envisager la question sur des territoires restreints dans lesquels le rôle des individus apparaîtra. Tous les élus du conseil général du Finistère, département où se trouve la moitié des établissements Diwan, ont voté une motion pour que les deux collèges de l'association «relèvent de sa responsabilité, au même titre que les 63 autres collèges publics » et demandent au gouvernement de «rechercher et mettre en œuvre toutes les solutions juridiques permettant la poursuite du processus d'intégration des écoles Diwan au service public». Qu'en est-il dans les autres conseils généraux de Bretagne? Peut-on définir le Finistère et même certaines communes, comme Carhaix, qui abrite l'une des écoles Diwan les plus revendicatives, ou Landerneau, qui a confié le suivi du dossier au conseiller municipal de l'Union démocratique bretonne ${ }^{8}$, comme des locomotives du mouvement pour la langue bretonne ? Le conseil municipal de Saint-Nazaire, en Loire-Atlantique, a reporté l'intégration de l'école Diwan. Quelle a été la teneur des débats, comment a été prise la décision? Les projets géopolitiques des personnes qui apprennent le breton en Loire-Atlantique sont-ils les mêmes que ceux des membres de Diwan dans le Finistère?

8. L'UDB est un parti régionaliste qui demande la reconnaissance du peuple breton (assortie d'une citoyenneté bretonne), une large autonomie respectant les frontières historiques de la Bretagne, la représentation directe du peuple breton dans les instances européennes, une Europe fédérale des peuples et des régions. 
EXTENSION DE L'AIRE LINGUISTIQUE BRETONNE EN PAYS GALLO

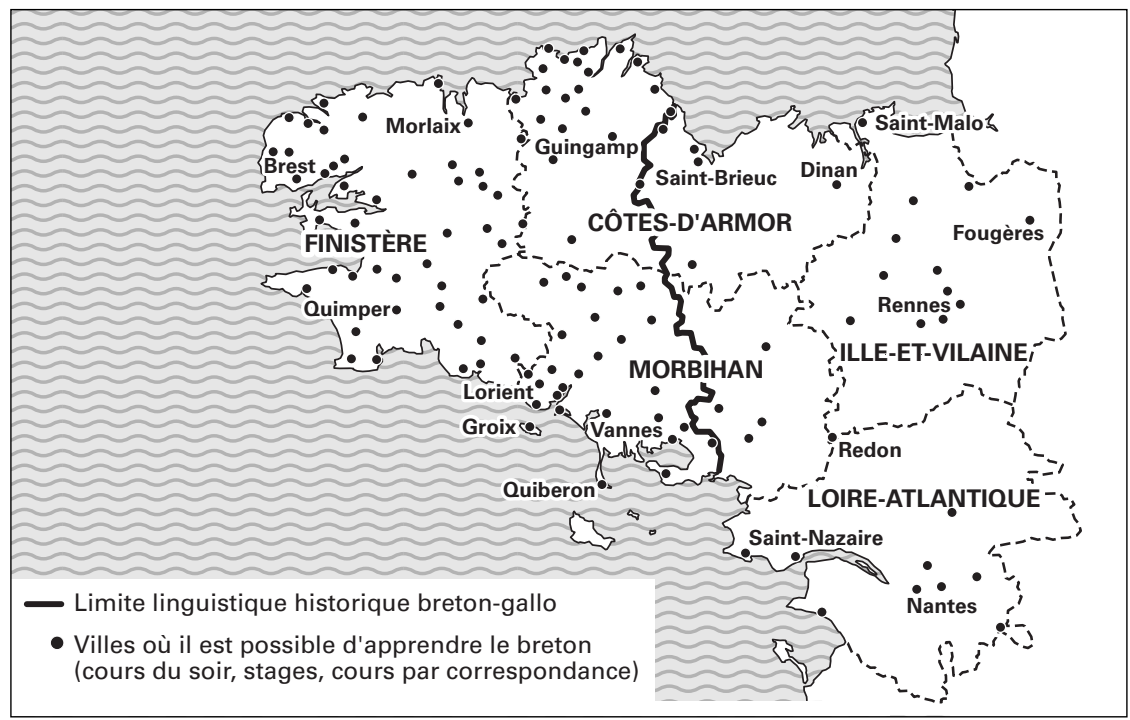

\section{TOPONYMIE BRETONNE EN PAYS GALLO}

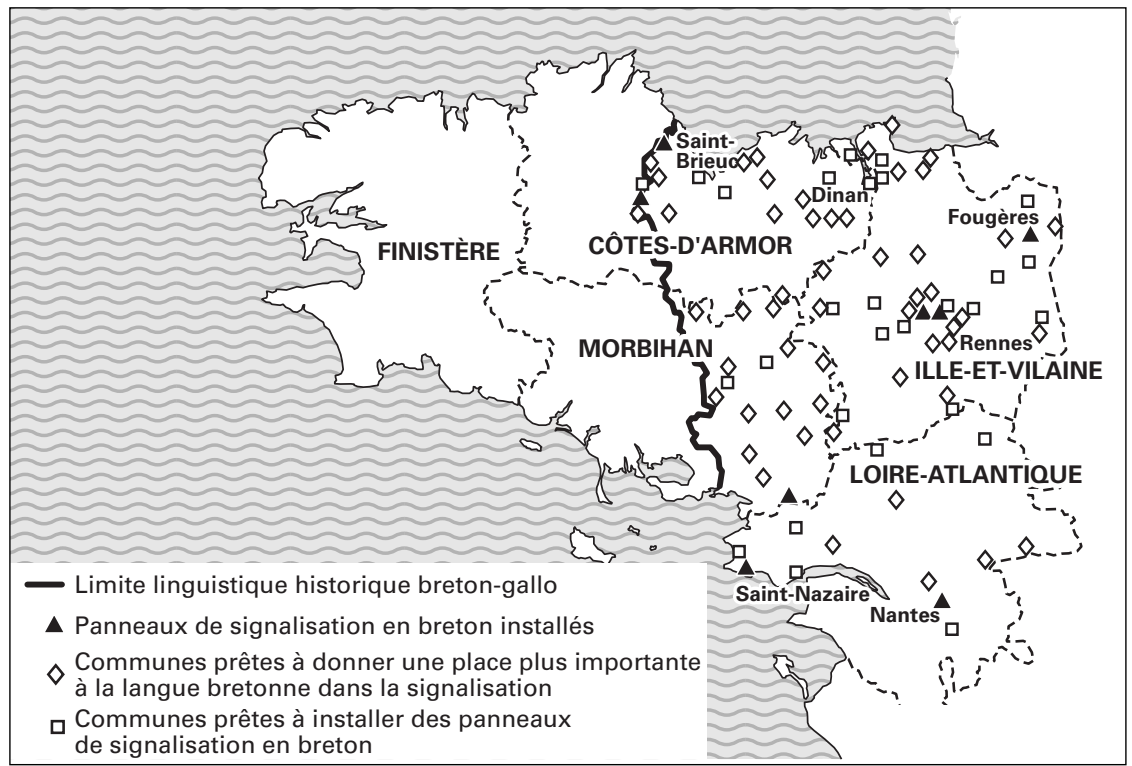


Au Pays basque, la commune de Hendaye (12 000 habitants) est un espace de dimension réduite dont l'évolution en matière linguistique est particulière. Comme c'est un cadre balnéaire enchanteur par rapport aux villes industrielles du Sud, notamment Irun, et parce que durant les années quatre-vingt les taux d'intérêt des banques françaises pour l'immobilier étaient plus avantageux que ceux d'Espagne, Hendaye accueille depuis vingt ans un nombre grandissant de résidents basques espagnols. Un tiers des enfants scolarisés ne parlent pas le français lorsqu'ils arrivent à l'école. Ils l'apprennent dans le système bilingue, mais restent dans un univers bascophone et hispanophone s'ils sont dans l'ikastola de Seaska.

Il est utile de concrétiser la spécificité de lieux aussi petits que des communes ou des quartiers, et de rappeler leur taille ou leur poids démographique par rapport à un ensemble plus vaste (département, région...), car c'est souvent dans des espaces restreints que se développent des contradictions qui ne sont pas forcément le souci de la majorité des habitants d'une région, mais occupent le devant de la scène. En retour, l'impact qu'ont de tels événements sur un ensemble spatial plus vaste - région, État, nation - dépend des représentations générales décrites plus haut. Si Juan Cruz Alli, l'ex-président navarrais, a surpris ses collègues régionalistes de l'UPN, c'est parce qu'il a adopté un autre espace symbolique de référence que la nation espagnole. Il a défendu l'idée que les États-nations actuels seraient bientôt caducs et qu'il fallait parier sur l'émergence d'une fédération des régions de l'Union européenne. Dans cette optique, l'alliance de la Navarre avec la communauté autonome basque lui apparaissait comme un atout. La méthode géopolitique explicite cette relation entre les représentations que les acteurs se font des territoires et la vie politique locale concrète, ainsi que leur évolution dans le temps.

\section{Le choix des représentations utiles aux idées que l'on poursuit}

Le fondateur et président d'Ikas Bi, Thierry Delobel, est un bon exemple de personnalité influente qui donne du sens à son action en l'intégrant dans des représentations géopolitiques plus générales. Le fait que François Bayrou, lorsqu'il était ministre de l'Éducation nationale, ait retiré des subventions à certains projets de l'association Ikas Bi a en effet amené Thierry Delobel à chercher de l'argent ailleurs. Il en a trouvé dans les arcanes de la communauté autonome basque. C'est le gouvernement de cette communauté autonome d'Espagne qui a subventionné une partie des manuels scolaires en euskara, visés par le ministère français de l'Éducation nationale ${ }^{9}$. De même, le centre de vacances en immersion linguis-

9. Aujourd'hui, les choses ont changé. Le Centre régional de documentation pédagogique (CRDP) donne sa validation pédagogique aux projets d'édition de matériel scolaire en euskara. 
tique d'Ascarat, ouvert en 1991 par Ikas Bi, a été renfloué par des fonds du Pays basque espagnol. La création de cet établissement avait été jugée nécessaire par l'association, car $80 \%$ des enfants des sections bilingues ne peuvent pas employer l'euskara en famille. Mais les enseignants de France n'ont pas utilisé cette possibilité d'immersion et les parents non plus. Ikas Bi s'est alors adressé au Pays basque sud pour recevoir des enfants et diminuer son déficit: en 2000, $70 \%$ des financements du centre venaient de la communauté autonome du Pays basque d'Espagne. Le président d'Ikas Bi a donc multiplié des contacts, qu'il dit chaleureux, avec les responsables de l'administration nationaliste basque. Cela a sans doute renforcé sa conviction que, dans le cadre de l'Union européenne et du développement des programmes transfrontaliers, le sentiment identitaire sera un des facteurs déterminants de la compétitivité des régions. C'est pourquoi le rapprochement avec les institutions du Pays basque d'Espagne, contrôlées par les nationalistes basques, lui semble souhaitable. Il dit «naturel». Un homme comme Thierry Delobel préfère se tourner vers l'administration nationaliste basque, au sud de la frontière, dont il ne craint pas de servir aussi les intérêts à long terme parce qu'ils rejoignent en partie les siens, plutôt que de dépendre de l'Aquitaine, qu'il juge lointaine et peu dynamique. De Bordeaux, dit-il, il n'y a rien à attendre. La région Aquitaine lui apparaît comme une entrave à ses projets, sans doute parce que, au sein de l'assemblée et des administrations régionales, ceux-ci ne sont portés par personne. Pour Thierry Delobel, les élites locales ont besoin de pouvoir «investir une institution». Cela rejoint la question du département Pays basque séparé du Béarn. Le département, que Delobel décrit comme une «meilleure courroie de transmission avec l'État», serait aussi un lieu de pouvoir dans lequel ses idées ont plus de chances d'être majoritaires. De son point de vue, «ce n'est pas grave que ce département puisse être pour les uns une fin en soi, et pour les autres une étape» vers l'union des sept provinces de la nation basque. Si une région basque transfrontalière voyait le jour, associant un département à la communauté autonome d'Espagne, il est clair qu'il y aurait beaucoup plus d'argent consacré à l'euskara (écoles, édition, traduction, radio, télévision, presse écrite...). Une partie de la population en verrait immédiatement le bénéfice. Le budget de la communauté autonome est dix fois supérieur à celui de la région Aquitaine ${ }^{10}$, qui a des compétences beaucoup moins étendues. Il est ainsi utile de mettre l'accent non seulement sur l'activité de telles personnalités, mais également

Cette validation est nécessaire à l'octroi par les collectivités territoriales françaises de subventions pour l'édition de ce matériel.

10. Budget 2000 de la communauté autonome basque: 5173761000 euros. Budget Aquitaine 2002: 660037 euros, soit un rapport de 1 à 8. 
sur les représentations qu'elles choisissent de privilégier, parfois consciemment, pour faire avancer leurs idées ${ }^{11}$.

Beaucoup de citoyens qui sont favorables aux langues régionales ne partagent pas pour autant les mêmes représentations de l'avenir. La dévolution de la politique linguistique à la région Aquitaine, ou bien à un département PyrénéesAtlantiques, ou encore à un hypothétique département basque, ébauche peut-être d'une future eurorégion basque, serait une décision géopolitique. Les arguments purement linguistiques ne doivent pas escamoter cet enjeu. L'identité culturelle d'un territoire est en effet un élément que l'on peut retrouver partout, qui semble «naturellement» tout influer. Mais rien n'est moins «naturel» qu'un territoire. Il est le résultat d'une patiente construction dans laquelle les symboles jouent un grand rôle. Dans le langage courant, on qualifie souvent de «symboliques» les actions ou paroles auxquelles on n'attache pas grand poids. Le mot est employé au sens de « très petite quantité ». Mais les symboles sont importants, car ils cristallisent des représentations historiques ou politiques nécessaires à l'action. C'est l'enjeu aussi de ce qu'on appelle la «territorialisation» des compétences linguistiques, c'est-à-dire le fait de donner aux instances régionales le pouvoir de légiférer sur l'enseignement ou l'usage social des langues régionales.

\section{La région : territoire du bilinguisme?}

Vers la région convergent des objectifs distincts. Les régionalistes convaincus ont besoin de l'argument culturel et historique pour détendre ou dissoudre le lien que l'histoire a forgé entre les États-nations dont ils dépendent et leur région. De leur côté, les partisans des «petites» langues ont besoin de cadres régionaux culturellement définis pour affermir l'usage social des langues minoritaires. L'opinion est en fait assez répandue que l'on assiste, comme le dit Jacques-André Tschoumy ${ }^{12}$, «à un tournant historique qui, de l'Europe des États-nations créée vers 1850, conduit à une cartographie toute nouvelle de l'Europe. Cette évolution sera marquée

11. Entretien, avril 2001. Thierry Delobel est aussi président de la Fédération pour les langues régionales dans l'enseignement public (FLAREP). Je ne lui ai pas demandé les raisons qui l'ont mené à s'engager si fortement pour la cause de l'euskara (il a payé de ses deniers la fabrication de la première brochure d'information). Fut-ce pour des raisons familiales, philosophiques, ou bien le fait d'avoir ouvert des cabinets dentaires à la fois en France et en Espagne (Pays basque, Rioja) lui a-t-il donné des idées sur les liens à développer de part et d'autre de la frontière? Les réponses éclaireraient sans doute certains de ses choix et compléteraient utilement l'analyse.

12. In Claude TRUCHOT (dir.), Le Plurilinguisme européen, Honoré Champion, Paris, 1994, p. 110. 
de la rupture d'une Europe dominée par les pays de grande culture, de l'essor de communautés politiques marginales, de la revanche des langues minoritaires et de l'avènement de pays "petits-moyens" mieux préparés à l'Europe de demain que les grandes nations qui ont façonné l'histoire jusqu'à présent [...]». C'est ce que défendait, d'une certaine manière, l'ancien président navarrais Juan Cruz Alli.

Mais l'idée que l'adéquation entre pouvoir et langue régionale résoudrait tous les problèmes évite d'aborder la question de la relation des personnes qui sont bilingues, «français-langue régionale», avec celles qui ne le sont pas. Que le territoire concerné soit celui d'un État ou d'une région ne change pas à mon sens la nature du problème. En effet, si d'un point de vue strictement linguistique il n'y a pas de hiérarchie entre les langues, ce n'est plus pareil dès que l'on envisage leur insertion dans des systèmes juridiques appliqués à un territoire, quel qu'il soit. Dans ce cas, il faut bien définir la relation qui s'établit entre les locuteurs des différentes langues. Les linguistes ont forgé le concept de «diglossie» pour décrire la coexistence entre deux variétés d'une même langue, aux fonctions ou statuts différents (espace privé/public, oral/écrit). Le mot s'applique à la différenciation que fait un locuteur bilingue dans l'usage des deux parlers, ou des deux langues. Il est en général utilisé de manière négative car les locuteurs des idiomes minoritaires finissent souvent par adopter les «grandes» langues, qui offrent plus d'opportunités sociales, et laissent les premières disparaître. D'autres linguistes jugent que le concept de diglossie n'est pas suffisant pour exprimer la rivalité entre des locuteurs de langues distinctes cohabitant sur un même territoire. Les Catalans ont notamment développé le concept de «conflit linguistique», qui décrit une situation dont l'enjeu est la substitution d'une langue par l'autre. Le mot «diglossie» désigne une «polarité sociolinguistique neutre» alors que celui de «conflit»s'applique à une "polarité problématique entre une langue dominante et une langue dominée » et traduit un dilemme: "Ou bien la langue imposée va se substituer lentement, mais sûrement, à la langue dominée, ou les usagers de celle-ci vont œuvrer à sa normalisation, c'est-à-dire à une utilisation normée (standardisée) dans tous les domaines de la communication, en combattant sans relâche les tendances à l'assimilation ${ }^{13}$.»

La notion de conflit linguistique entre en contradiction avec celle de bilinguisme. «Les forces ultranationalistes et indépendantistes catalanes, écrit le sociolinguiste Gentil Puig i Moreno, sont fermement opposées au bilinguisme de masse, au concept lui-même [...]. Ils considèrent que le bilinguisme est une capitulation pour la langue catalane et que celle-ci ne peut retrouver un usage et une place hégémoniques qu'en redevenant langue première dans tous les domaines ${ }^{14}$. »

13. Henri Boyer, 1987, cité par Eva VetTer, Plus de breton?, Éditions An Here, Le Relecq-Kerhuon, 1999, p. 28.

14. «Problèmes de glottopolitique », Cahiers de linguistique sociale, $\mathrm{n}^{\circ}$ 7, 1985, p. 83. 
De la sociolinguistique catalane est sortie la mise en pratique de cette résistance face à l'influence du castillan. Selon le directeur général de la politique linguistique du gouvernement autonome de Catalogne, Lluis Jou, nationaliste catalan, «l'universalité de la connaissance [du catalan], qui évite la subordination linguistique, la double communication systématique et le stérile bilinguisme de façade, est la condition première de la survie [pervivencia] du catalan; le maintien d'espaces où son usage est en principe exclusif [espacios de uso normalmente exclusivo], ainsi que l'obtention de sa présence significative dans d'autres champs, est l'unique garantie d'utilité sociale et la condition incontournable pour éviter sa dépendance vis-à-vis du castillan [supeditación al castellano] ». L'idée est que la langue catalane risque à terme d'être marginalisée, voire de disparaître, si elle n'acquiert pas une forme de prééminence dans la communauté autonome de Catalogne («elle doit être indispensable dans certains secteurs, comme l'éducation, l'administration ou les services publics »). Cette conviction se fonde sur une comparaison à l'échelle de l'Espagne et du monde, qui met en valeur la domination du castillan, parlé par 400 millions de personnes contre 10 millions de catalanophones. La communauté autonome est l'«espace vital» du catalan, dans lequel il doit être la «langue de tous, d'identification collective et d'intégration sociale». «Le catalan doit se voir garantir en Catalogne le respect, le prestige et l'utilité sociale ainsi qu' un usage complet [la plenitud de uso ${ }^{15}$.»

C'est de l'inversion de la hiérarchie linguistique à l'intérieur de la communauté autonome dont il est question ici, et non d'une égalité des langues, qui est perçue comme un leurre. Lorsque l'on réclame, comme en Corse, la co-officialité de deux langues dans une région, ce qui est un terme désignant une relation politique entre elles, se place-t-on dans l'optique que prônent les catalanistes cités plus haut?

Roland Breton, géographe engagé pour les langues minoritaires ${ }^{16}$, distingue les langues «régionales », dont l'extension territoriale n'est pas légalement définie, et les langues «territoriales », qui ont un statut particulier au sein d'une région (comme le corse). De son point de vue, seule la généralisation du statut de langue territoriale permettra d'organiser la «survie des langues dans l'égalité ». Cela signifie que pour mettre deux langues, nationale et régionale, sur un pied d'égalité, il faut donner des compétences aux régions en matière linguistique. "La situation linguistique particulièrement complexe de l'Europe, poursuit cet auteur, et l'avancement culturel de la plupart de ses composantes ethniques lui imposent de rechercher des formules qui devraient cesser définitivement d'asseoir la grandeur de certains ensembles humains sur l'abaissement des autres considérés comme mineurs et négligeables. » Présentée de cette façon, la survie des langues

15. El País, édition Barcelone, 15 janvier 2002.

16. Voir notamment son article «Linguicide à l'école, crimes d'État», publié dans Le Monde de l'éducation et dans la revue Fédéchoses, «Pour le fédéralisme», $3^{\mathrm{e}}$ trimestre 2001, n 113. 
dans l'égalité est étroitement liée à l'évolution des hiérarchies entre le pouvoir régional et celui de l'État.

Pourtant, organiser dans l'harmonie une société dont les habitants parlent les deux langues du territoire ne dépend pas seulement de la décentralisation des pouvoirs. Ce bilinguisme est si difficile à organiser qu'il n'existe pratiquement pas à l'heure actuelle en Europe ${ }^{17}$, sauf peut-être en Catalogne, qui constitue un cas un peu spécial. Les nationalistes et les non-nationalistes catalans parlent et défendent tous la langue catalane. Si les socialistes gagnent les élections, rien ne changera sur ce plan. C'est dû à l'histoire, mais également à la proximité linguistique entre le catalan et le castillan. Celui qui parle castillan (ou français) peut lire un texte simple en catalan, le comprendre si son interlocuteur le veut bien, et l'apprendre sans trop de peine. Les catalanistes peuvent être radicaux sans affecter profondément la vie de leurs concitoyens. C'est différent dans le cas du basque par rapport au français et à l'espagnol, du breton ou du flamand par rapport au français, du gaélique par rapport à l'anglais.

Au Pays basque, le principal problème pour développer une société bilingue réside non seulement dans la difficulté que représente l'apprentissage du basque pour un adulte, mais aussi dans le lien entre langue et politique. Pour les nationalistes basques, la connaissance de l'euskara devrait avoir un caractère obligatoire dans la communauté autonome au même titre que l'espagnol. C'est une question de souveraineté linguistique. D'un autre côté, la plupart des hommes politiques non nationalistes basques ne parlent pas ou parlent mal l'euskara. C'est surtout le cas au sein du Parti populaire. Pour le Parti socialiste, l'intégration du parti nationaliste Euskadiko Ezkerra a augmenté la proportion de bascophones, mais elle reste faible. Au Guipuzcoa, on compte 15 bascophones sur 96 élus ${ }^{18}$ (la population de bascophones étant évaluée à $50 \%$ du total provincial). La division entre les locuteurs bilingues euskara-espagnol et les autres Basques est très forte. Même chez les jeunes adultes, le système fomente une séparation puisque l'on doit choisir de faire ses études universitaires soit tout en euskara, soit tout en espagnol. Et la situation ne va pas s'améliorer à court terme : le contexte de terreur actuel crée un climat de moins en moins favorable au bilinguisme.

Le lien entre région et langue régionale est souvent montré comme allant de soi, alors qu'on a vu que la coïncidence entre langue bretonne et Bretagne, ou entre langue basque et Pays basque, n'est pas une réalité linguistique mais une représentation historique ou politique. Ce lien est aussi proposé comme la solution à toutes les difficultés, alors qu'on a vu qu'il peut en faire surgir d'autres. Il faut bien préciser les enjeux des revendications d'égalité des langues dans leurs

17. En Suisse et en Belgique, sauf exception, les territoires sont monolingues. Voir Paul TOURRET, « Nationalismes régionaux en Europe », Hérodote ${ }^{\circ}$ 95, $4^{\mathrm{e}}$ trimestre 1999.

18. Douze conseillers municipaux sur 86,2 conseillers provinciaux sur 10 , et 1 député au parlement autonome sur 4. (Source personnelle.) 
contextes géopolitiques particuliers. L'histoire des langues détermine en partie le pouvoir de zizanie qu'elles contiennent. Il est très important de garder à l'esprit cette diversité pour comprendre qu'une politique linguistique comparable peut avoir des effets différents selon les situations géopolitiques. Le gaélique ne représentera pas la même chose en république d'Irlande que dans l'Ulster, qui connaît une évolution rapide des rapports de forces entre nationalistes irlandais et unionistes. En Alsace, le programme de développement de l'enseignement bilingue fait de l'allemand la langue régionale en se fondant sur les définitions proposées par l'université Marc-Bloch de Strasbourg: «Langue écrite et langue de référence des dialectes alémaniques et franciques parlés en Alsace - et dans une partie de la Moselle -, l'allemand est une langue régionale de France» (juin 1985). «L'allemand présente en effet, du point de vue éducatif, la triple vertu d'être à la fois la langue écrite et la langue de référence des dialectes régionaux, la langue des pays les plus voisins et une grande langue de diffusion européenne et internationale » (septembre 1991). Cette option peut aboutir à la disparition de l'alsacien. On mesure combien le choix de ce bilinguisme est différent de celui de la Bretagne ou du Pays basque. Le débat en France n'est pas dans «une impasse» comme l'écrit Henri Giordan, il met simplement à jour les nombreuses contradictions de la question linguistique et ses liens complexes avec la politique.

\section{La relation entre le français et les langues régionales}

Le problème de l'intégration ou non des écoles Diwan dans le secteur public est compliqué parce que, entre autres choses, il est difficile de mesurer l'impact que pourrait avoir la généralisation d'une telle mesure à des régions et situations très variées. Depuis 1994, Diwan bénéficie, comme Seaska, du statut d'établissement privé sous contrat. Le personnel y est payé par l'État mais pas les investissements d'équipement (locaux, matériel). En vertu de la loi Falloux de 1850, ces écoles ne peuvent être subventionnées par les collectivités locales qu'à hauteur de $10 \%$ de l'investissement engagé, parce que ce sont des établissements privés. En mai 2001, un protocole d'accord a été signé avec le ministère de Jack Lang pour l'intégration de Diwan dans le service public, mais cette intégration a depuis été suspendue par le Conseil d'État le 30 octobre 2001, au motif que Diwan pratique une méthode d'immersion totale.

D'un point de vue politique, la controverse n'oppose pas un État à une société en mal d'identité, ou à des régions, mais des citoyens qui ne sont pas d'accord entre eux. L'ordonnance du 30 octobre répond à une requête déposée par le Syndicat de l'enseignement du second degré (SNES), l'Union nationale des syndicats autonomes (UNSA), la Ligue de l'enseignement, la Fédération des conseils de parents d'élèves des écoles publiques (FCPE), la Fédération des délégués départementaux de l'Éducation nationale. Ces requêtes concernaient à la fois l'enseigne- 
ment bilingue et l'immersion totale. Le SNES dénonce que «le ministre feint de confondre enseignement "des langues régionales" et enseignement "en langues régionales"». L'UNSA, la Ligue de l'enseignement, la FCPE soutiennent que «la méthode d'enseignement en langue régionale par immersion préjudicie [...] à un intérêt public ; [...] elle met en cause l'unité de la République». En réponse à ces requêtes, le Conseil d'État n'a suspendu l'exécution que «des actes contestés en tant qu'ils concernent l'enseignement bilingue par la méthode de "l'immersion"».

En Bretagne, le débat divise la population. Les parents de la FCPE du lycée Jean-Marie-Le-Bris de Douarnenez ont écrit, par exemple, que «la pédagogie par immersion ne menace en rien la langue française ni les valeurs de la République, et cela ouvre des perspectives d'innovation et d'expérimentation pédagogique, y compris pour l'apprentissage des langues étrangères, domaine dans lequel la France est loin d'atteindre la performance ${ }^{19}{ }^{»}$. Jackie Guigo, du Mouvement des citoyens de Douarnenez, répondait quelques jours plus tard qu'admettre le principe que l'école publique puisse se dérouler sans langue française, même en maternelle, c'est créer « des sous-communautés identitaires qui [...] essaient à tour de rôle d'imposer des droits qui leur seraient spécifiques ${ }^{20} »$. En outre, ajoutait-il, rien n'empêchera à la suite d'une telle décision «les locuteurs des langues de l'immigration de demander la même chose, ce qui suppose une mobilisation considérable de moyens dirigés vers l'apprentissage d'autres langues que le français». Les parents FCPE du lycée Jean-Marie-Le-Bris écrivent qu'ils ont un «doute sur les motivations profondes du recours» au Conseil d'État, sans doute pour suggérer que ces motivations sont politiques. Et en effet elles le sont, parce que c'est une question qui dépasse la linguistique. Andrew Lincoln, président des écoles Diwan, pense lui aussi qu'il s'agit de politique. La question doit maintenant être jugée sur le fond. «Il ne s'agit pas, dit-il, d'attendre passivement, il faut préparer une réaction très forte sur ces questions qui soulèvent la fragilité de tous les acquis sur les langues régionales [...]. Il faut décider une fois pour toutes, faut-il aller vers des changements législatifs et constitutionnels pour parvenir à cette intégration ${ }^{21}$ ?»

La polémique n'est pas seulement pédagogique. Elle se rapporte aux relations que l'on veut établir entre le français et les langues régionales. En Bretagne, l'Association des parents d'élèves pour l'enseignement en breton dans l'école publique se différencie de Diwan par la place qu'elle accorde au français «toujours présent depuis la maternelle». «C'est un projet parental. On donne tous les outils à l'enfant pour qu'il puisse être bilingue, il peut choisir ${ }^{22}$. »C'est aussi la

19. Le Télégramme, 28 janvier 2001.

20. Le Télégramme, 31 janvier 2001.

21. Le Télégramme, 31 octobre 2001.

22. Entretien Judith Castel, Div Yezh, Rennes, mars 2002. 
raison pour laquelle Div Yezh préfère essaimer des classes bilingues dans toutes les écoles, afin de créer une «offre de choix pédagogique aux familles». L'association ne souhaite pas concentrer les ouvertures de ces classes sur un petit nombre d'établissements que les parents pourraient assimiler à des centres d'enseignement en breton différents du reste des écoles.

La controverse sur l'immersion et la place du français se développe aussi au Pays basque français, mais d'une manière un peu différente. Tout d'abord, au sein des écoles avec sections bilingues, les conseils des maîtres ont tous opté pour un système dans lequel une classe est composée d'enfants pratiquant l'euskara et d'autres qui ne le font pas. À certaines heures, la classe se divise pour rejoindre deux cours différents, l'un en français, l'autre en euskara. Cela oblige le professeur en euskara à travailler en concertation avec de nombreux collègues puisque son cours réunit des enfants qui viennent de plusieurs classes. Ce choix vise à éviter la clôture des enfants sur un milieu scolaire bilingue qui percevrait trop nettement sa différence, une sorte de "ghetto» bilingue. Deux écoles ont, dans le passé, regroupé les élèves de la section bilingue dans des classes spéciales. Cela simplifie le travail du professeur en euskara, qui ne doit se concerter qu'avec un seul collègue, celui qui assure les cours que toute la classe suit en français. Mais, même dans ce cas, des activités en commun réunissaient de temps à autre l'ensemble des élèves. Aujourd'hui, Thierry Delobel demande que l'immersion totale soit étendue aux petites classes des sections actuellement bilingues de l'école publique, ce qui ne fait pas l'unanimité dans Ikas Bi. Un récent sondage réalisé sur la communauté d'agglomération Bayonne-Anglet-Biarritz, la zone la plus densément peuplée du département des Pyrénées-Atlantiques, a d'ailleurs fait apparaître que la majorité des parents souhaitant l'enseignement du basque opteraient pour le bilinguisme et non pour l'immersion totale ${ }^{23}$. Enfin, de son côté, l'asso-

23. Sondage CSA (synthèse des principaux enseignements) auprès de cinq cents parents d'enfants de zéro à deux ans, en octobre 2001 : $19 \%$ des parents souhaitent «tout à fait » et $37 \%$ «plutôt» que leur enfant apprenne l'euskara, soit $56 \%$ de réponses positives (64\% chez les parents âgés de moins de trente ans).

La majorité des parents qui souhaitent que leur enfant apprenne le basque à la maternelle déclarent également souhaiter qu'il maîtrise parfaitement le français et ait une connaissance suffisante du basque pour le comprendre et le parler. $81 \%$ de ces parents optent pour le modèle pédagogique consistant en un enseignement dispensé pour moitié en basque et pour moitié en français en maternelle et en élémentaire. $13 \%$ seulement optent pour un enseignement dispensé en basque en maternelle avec introduction progressive du français à l'issue de la maternelle.

Les motivations des parents qui souhaitent l'enseignement de l'euskara sont variées : l'attachement aux traditions, au patrimoine, aux racines est cité par $17 \%$ des sondés (dont $20 \%$ des parents nés au Pays basque). $37 \%$ des interviewés qui souhaitent que leur enfant apprenne le basque à l'école invoquent comme raison le fait de vivre au Pays basque. La culture, l'ouverture d'esprit et l'enrichissement sont mentionnés par $24 \%$ d'entre eux. Concernant les $44 \%$ qui ne souhaitent pas que leur enfant apprenne la langue basque à l'école, l'hostilité de principe à 
ciation Seaska a refusé les propositions d'intégration au secteur public que lui a faites le ministre de l'Éducation nationale. L'association argue qu'en son sein les parents participent à l'élaboration de la pédagogie, ce qui ne pourrait plus être le cas dans le secteur public. Une partie du problème réside sans doute aussi dans les relations des nationalistes basques d'Espagne avec Seaska. Jusqu'à l'année dernière, elle recevait quelque 3,5 millions de francs par an de la part du gouvernement basque et on ne sait pas combien de la part d'Udalbitza, l'assemblée des communes nationalistes basques, financée par le même gouvernement autonome. Dans ce contexte d'un territoire revendiqué par deux nations, la basque et la française, la question de la relation à la langue de l'État est plus politique encore qu'ailleurs.

Pour l'instant, les autorités locales semblent plutôt vouloir éviter de créer un conflit au sujet de l'euskara, sans doute parce que l'opinion publique reçoit avec bienveillance tout ce qui favorise la langue basque. Une bataille à ce sujet est perçue par une bonne partie de la classe politique comme un jeu où qui perd gagne. L'impossibilité légale de financer les bâtiments de Seaska a déjà été contournée par Hendaye, Biarritz, Urrugne et Bayonne, au moyen d'arguties réglementaires pour désamorcer les remarques de la chambre régionale des comptes. Un collège de Seaska est actuellement en trop mauvais état pour continuer à accueillir des élèves à la rentrée prochaine. Avec son refus d'intégrer le système public, Seaska portait la responsabilité de ne pas avoir de locaux aux normes. L'arrêt du Conseil d'État va rejeter théoriquement l'accusation d'intransigeance sur le ministère de l'Éducation nationale, ou sur le département, qui appliquera la loi Falloux.

La relation de la langue régionale au français est une affaire pratique : lors d'un symposium entre spécialistes des politiques linguistiques, Christiane Marcellesi, épouse du sociolinguiste corse Jean-Baptiste Marcellesi, disait par exemple au sujet de l'enseignement en occitan: "Quel rôle et quelle place donnez-vous au français lorsque vous revendiquez pour l'occitan le statut de langue enseignante? Il me semble que c'est bel et bien le français qui devient une langue minorée. Refusez-vous le rôle national du français ? Quelle place, dans un tel cadre, donnezvous au français dans la vie économique, scientifique, etc. nationale et internationale ? Que faire des réalités du marché de l'emploi, des nécessités et réalités de la mobilité de l'emploi ? Voulez-vous réenfermer les enfants dans le ghetto du monolinguisme régional-social ? [...] Pour moi, conclut-elle, il s'agit en priorité de défendre à la fois un bilinguisme national-régional et un multilinguisme social de masse ${ }^{24}$.»

Mais, comme le fait apparaître aussi la question «Refusez-vous le rôle national du français?», cette relation est également symbolique. La nation française

fondement idéologique ne se manifeste pas dans les réponses spontanées des interviewés: seuls $4 \%$ d'entre eux évoquent un enseignement trop «politisé ». C'est à Biarritz que cette méfiance se manifeste le plus.

24. «Problèmes de glottopolitique», Cahiers de linguistique sociale, $\mathrm{n}^{\circ}$ 7, 1985, p. 71. 
comme la nation espagnole sont aussi des représentations, qui se trouvent consolidées ou fragilisées par le statut accordé à la langue nationale par rapport aux langues régionales. L'hypothèse que je formule ici est que la revendication d'égalité des langues dans tous les domaines, c'est-à-dire le refus de relations hiérarchiques entre les langues, le refus qu'une langue ait le monopole de certaines fonctions, a de forts risques de déboucher sur une contestation des relations de pouvoir établies au sein d'un État, notamment entre l'État et les régions. Rappelons qu'après l'enseignement les politiques linguistiques concernent les médias, les activités culturelles, la vie économique et sociale, les services publics et la justice. Le statut accordé à une langue n'est pas seulement juridique. C'est une opinion subjective à son sujet, qui reflète aussi un état des rapports sociaux. Lorsque le président du Parti nationaliste basque, Xavier Arzalluz, dit que l'espagnol est la «langue de Franco », j'en déduis qu'il a beau admettre la loi l'obligeant à reconnaître les droits des non-bascophones, son utopie est d'imposer un jour la seule langue nationale qui vaille à ses yeux, l'euskara, parce qu'il conteste la souveraineté espagnole sur sa nation basque. On entendra aussi dire, pour affirmer un désir de revanche des langues régionales, que le français est la langue des tortionnaires d'Algérie.

Une langue, explique Philippe Blanchet, peut avoir un statut bas et une pratique importante, un statut élevé et une faible pratique, ou bien conjuguer les deux paramètres. Il paraît aller de soi que cette distinction ne concerne que les langues minoritaires, surtout en France, où la domination du français est écrasante. Pourtant, il me semble qu'un débat sur le développement de pratiques linguistiques diversifiées dans notre pays ne devrait pas faire l'économie d'une réflexion sur le statut que les citoyens de ce pays accordent à la langue nationale.

\section{Une substitution des élites?}

La question linguistique ne concerne donc pas que l'enseignement car les langues ont des fonctions multiples. Dans diverses communautés autonomes d'Espagne, le statut de langues co-officielles a ouvert une part non négligeable de l'emploi aux personnes bilingues espagnol-«langue propre» (c'est ainsi que l'on nomme les autres idiomes). Dans la communauté basque, du fait de la dimension symbolique et politique de l'euskara, la mise en place d'une société bilingue s'est accompagnée d'un phénomène que l'on pourrait qualifier de substitution des élites car la minorité des personnes vraiment bilingues euskara-espagnol ${ }^{25} \mathrm{~s}$ 'est trouvée en position de prendre les rênes d'institutions nouvelles (le premier gouvernement autonome se met en place en 1980) pour lesquelles cette compétence

25. En 1986, 24,5\% de la population se déclaraient bascophones, $15 \%$ seulement sachant lire et écrire la langue. 57,9\% ne parlaient pas du tout le basque (entre les deux, il y a une catégorie de «presque bascophones »), Anuario Estadístico Vasco, Eustat, 1988. 
était requise. Certains socialistes pensent qu'ils ont alors fait preuve d'une trop grande complaisance envers les défenseurs de l'euskara, car cela a contribué à affaiblir l'influence de leurs idées politiques au profit de celles des adversaires nationalistes. En 1986, le PSOE a, par exemple, laissé le PNV prendre la présidence du gouvernement alors que, du fait d'une scission interne, ce dernier s'était retrouvé en deuxième position derrière les socialistes. Cette décision fut prise pour accéder à des postes de responsabilité tout en évitant les blocages que les partis nationalistes ne manqueraient pas de provoquer au parlement. L'un des protagonistes de cette époque, Ramón Recalde ${ }^{26}$, croit aujourd'hui, à la lumière de l'évolution catastrophique de la situation, que les socialistes se sont alors trompés et qu'ils ont été «utilisés par les nationalistes pour augmenter leur hégémonie». Ramón Recalde attribue cette «erreur » à un «sentiment de mauvaise conscience» à cause du nationalisme espagnol de Franco, dont ils n'étaient pourtant pas les héritiers. «Nous nous sentions d'une certaine façon disqualifiés et il fallait accepter le plus du nationalisme. Cela [...] a contribué à étendre cette façon de penser basique du nationalisme qu'il est très difficile de vaincre ${ }^{27}$.»

En Navarre, dans les institutions régionales, le modus vivendi des bascophones et des non-bascophones tient sur le mutisme, le non-dit. On ne connaît pas les convictions de tel ou tel directeur de cabinet lié aux associations militantes de l'euskara. Le soupçon y tient lieu de débat, et toute décision, si minime soit-elle, en faveur ou contre le développement de l'euskara est perçue comme une victoire ou une défaite selon les camps.

La suspicion envers l'extension d'une langue minoritaire sur un territoire prend racine dans la crainte que cela puisse changer les rapports de forces existants, notamment parmi les élites. On entend souvent dire, en effet, qu'une langue minoritaire ne peut pas résister à la domination d'une grande langue de communication si les locuteurs ne peuvent l'utiliser dans leur vie sociale. Il faudrait, pour en justifier aussi l'enseignement, lui donner une place dans l'administration et, si possible, dans la vie économique, aux côtés de la langue nationale. Mais la discrimination positive pour les locuteurs d'une langue au sein de l'administration est un moyen pour ces derniers d'accéder à des postes où se prennent des décisions, ainsi qu'aux places de professeur, journaliste, directeur administratif, syndicaliste..., ce que j'appelle l'élite, d'où se diffusent les idées ${ }^{28}$. La réalité sociale qui

26. Un des promoteurs de l'enseignement du basque à la fin du franquisme, puis conseiller à l'éducation et à la justice dans la communauté autonome. L'ETA a tenté de le tuer en septembre 2000 .

27. Entretien publié dans la revue dominicale d'El País de septembre 2000, après l'attentat contre lui.

28. On m'a rapporté l'anecdote suivante: lors d'une exposition internationale au Japon, la Navarre était la seule région d'Espagne à avoir un stand, mais on n'y voyait que le drapeau basque, et les traditions présentées étaient celles de la montagne. 
en découle est liée à la proportion de bilingues dans la langue régionale, ainsi qu'aux flux migratoires internes à l'État, certaines zones attirant plus de populations nouvelles que d'autres. La portée de l'enjeu est fonction de l'idéologie politique (nationalisme, régionalisme...) associée, ou non, à la langue régionale.

D'un point de vue pratique, le choix entre la contrainte et le volontariat pour amener les citoyens à avoir besoin de la langue minoritaire dépend d'un cadre législatif, donc des résultats électoraux, mais aussi des rapports de forces au sein d'instances diverses. Le «dosage entre persuasion et coercition, écrit un spécialiste du Québec ${ }^{29}$, [se fait] à la suite de négociations et d'alliances avec les principaux agents d'application de la politique linguistique: les chefs d'entreprise, les syndicats, les groupes sociaux, les leaders des communautés linguistiques, les sociétés étrangères qui sont des partenaires commerciaux». Aujourd'hui, en Europe, la réussite ou l'échec d'un projet de diffusion linguistique ne reflète pas la victoire d'une langue innocente sur une langue de l'oppression, mais le résultat positif ou négatif d'un travail d'influence auprès de ces «principaux agents ». C'est ce travail qui aboutit à des décisions favorisant, ou freinant, l'extension des lieux d'usage social d'une langue minoritaire. D'où l'utilité d'occuper, ou de conserver si l'on n'est pas favorable à une telle diffusion, des lieux de pouvoir et d'influence très diversifiés. C'est de la politique.

Il est important que le débat sur les langues n'oppose pas une représentation de l'État inamovible face aux locuteurs de langues moins répandues qui seraient impuissants. Non seulement cette vision n'est pas juste, mais elle nourrit les discours légitimant l'usage de la violence et de l'intimidation contre les symboles et les représentants de l'État que l'on dit «criminel», ou même envers des concitoyens. En France, cela se passe en Corse depuis plus de vingt ans, et cela commence au Pays basque. La distance est si grande entre les militants ou les élus qui intimident leurs adversaires pour obtenir un résultat et ceux qui agissent dans le respect de la liberté d'opinion que les uns et les autres semblent ne pas pouvoir relever de la même réflexion. Dès l'instant où l'on est non violent, il n'y aurait pas de conflit. Mais l'analyse géopolitique s'applique à toutes sortes de rivalités, et notamment celles qui se déroulent dans le cadre du consensus démocratique. Dans un système démocratique, en effet, le pouvoir est multiforme, car on peut avoir de l'influence à partir d'une association, d'un journal, d'un syndicat. La description des stratégies mises en œuvre pour se trouver aux places où se prennent les décisions et à partir desquelles se diffusent des représentations géopolitiques est une information nécessaire à la réflexion d'ensemble.

29. Pierre-Étienne LAPORTE, président du Conseil de la langue française, Québec, in Claude TRUChOt (dir.), Le Plurilinguisme européen, op. cit., p. 107. 
La question linguistique est associée à celle de l'évolution des pouvoirs dans l'Union européenne. L'image irénique qui en est donnée par le Conseil de l'Europe vise à obtenir un résultat quantitatif, une augmentation du nombre de locuteurs. Mais elle ne permet pas d'aborder la nature des relations qui s'établissent entre ces locuteurs. Des idées simplistes et culpabilisantes sur les méfaits des États actuels auront comme principal résultat de conférer une portée symbolique supérieure aux victoires des uns et des autres, en laissant notamment plus de latitude aux groupes, pas forcément représentatifs, qui manipulent sciemment ces représentations afin d'imposer leur vision des choses. Les combats linguistiques se développent selon des modalités variées, et ces modes d'action, ainsi que les projets politiques qui s'y rattachent, peuvent et doivent être finement analysés pour être débattus. 